\title{
Nuclear translocation of glutathione transferase omega is a progression marker in Barrett's esophagus
}

\author{
SIMONA PIAGGI ${ }^{1}$, SANTINO MARCHI $^{2}$, EUGENIO CIANCIA ${ }^{3}$, NICOLA DEBORTOLI ${ }^{2}$, \\ ALESSANDRA LAZZAROTTI $^{1}$, MICHELA SAVIOZZI ${ }^{1}$, CHIARA RAGGI $^{1}$, VANNA FIERABRACCI $^{1}$, \\ ATHANASE VISVIKIS $^{4}$, HANNE C. BISGAARD ${ }^{5}$, ALESSANDRO F. CASINI ${ }^{1}$ and ALDO PAOLICCHI ${ }^{1}$ \\ ${ }^{1}$ Dipartimento di Patologia Sperimentale, Biotecnologie Mediche, Infettivologia ed Epidemiologia, Sezione \\ di Patologia Generale, ${ }^{2}$ Dipartimento di Medicina Interna, Università di Pisa; ${ }^{3}$ Azienda Ospedaliera Universitaria \\ Pisana, Pisa, Italy; ${ }^{4}$ Centre du Médicament, Université Henri Poincaré, Nancy, France; ${ }^{5}$ Department of Medical \\ Biochemistry and Genetics, The Panum Institute, University of Copenhagen, Copenhagen, Denmark
}

Received June 2, 2008; Accepted September 9, 2008

DOI: $10.3892 /$ or_00000219

\begin{abstract}
Barrett's esophagus (BE) represents a major risk factor for esophageal adenocarcinoma (AC). For this reason, patients with $\mathrm{BE}$ are subjected to a systematic endoscopic surveillance to detect initial evolution towards non-invasive neoplasia (NiN) and cancer, that eventually occurs only in a small fraction of BE patients. This study was aimed to investigate the possible role of glutathione-S-transferaseomega 1 (GSTO1), a recently discovered member of the glutathione-S-transferase family, as a progression marker in the Barrett's disease in order to improve the diagnosis of $\mathrm{NiN}$ in BE and to understand the mechanisms of the progression from $\mathrm{BE}$ to $\mathrm{AC}$. We investigated the expression and subcellular localization of GSTO1 in biopsies from patients with $\mathrm{BE}$ and in human cancer cell lines subjected to heath shock treatment. A selective nuclear localisation of GSTO1 was found in 16/16 biopsies with low- or highgrade $\mathrm{NiN}$, while it appeared in only $4 / 22 \mathrm{BE}$ biopsies without signs of $\mathrm{NiN}(\mathrm{P}<0.0001)$. Among biopsies of $\mathrm{BE}$ without $\mathrm{NiN}$, diffuse (nuclear and cytoplasmic) staining was found in $5 / 22$ cases, while selective cytoplasmic localisation was found in 13/22. The 6 cases with indefinite grade of NiN were equally divided between nuclear, cytoplasmic and diffuse staining (2 each, respectively). Experiments in vitro
\end{abstract}

Correspondence to: Dr Simona Piaggi, Dipartimento di Patologia Sperimentale, BMIE, Sezione Patologia Generale, via Roma 55, Pisa, Italy

E-mail: s.piaggi@med.unipi.it

Abbreviations: BE, Barrett's esophagus; AC, adenocarcinoma; NiN, non-invasive neoplasia; LG-NiN, low-grade NiN; HG-NiN, high-grade NiN; GSTO, glutathione-S-transferase-omega; GERD, gastroesophageal reflux disease; Hp, Helicobacter pylori

Key words: glutathione transferase, Barrett's esophagus showed that in human HeLa cancer cells, GSTO1 translocates into the nucleus as a consequence of heath shock. These findings suggested that the nuclear translocation of glutathione-S-transferase-omega 1 could be involved in the stress response of human cells playing a role in the cancer progression of Barrett's esophagus. Its immunohistochemical detection could represent a useful tool in the grading of Barrett's disease.

\section{Introduction}

Barrett's esophagus (BE), i.e., the presence of intestinal metaplasia and goblet cells in biopsies from lower esophagus, is observed in $5-15 \%$ of individuals with chronic gastroesophageal reflux disease $(1,2)$, and represents the main risk factor for adenocarcinoma (AC) of the esophagus $(3,4) \mathrm{AC}$ of the esophagus may develop through stages from metaplasia to increasing grade of non-invasive neoplasia (NiN) (low- and high-grade $\mathrm{NiN}$ ) (5).

The mechanisms leading to the substitution of squamous epithelium by specialized intestinal epithelium are poorly understood, but it is commonly accepted that Barrett esophagus follows the insufficiency of defence mechanisms of the esophageal mucosa (luminal secretion of mucus, bicarbonate, growth factors, etc.) to cope with repeated mucosal injury (3). Hydrogen ion, pepsin, trypsin and bile acids invade the esophageal mucosa as a consequence of gastresophageal reflux, and are thought to act synergistically to harm the epithelium of the lower part of the esophagus to determine the intestinal metaplasia (6).

The same agents that cause metaplasia are thought to act synergistically with carcinogens to cause cancer progression that leads to $\mathrm{NiN}$ and overt adenocarcinoma of the esophagus (6). Factors determining the survival of proliferating cells and their ability to withstand the hostile environment of the Barrett esophagus are thus likely to play a crucial role in esophageal carcinogenesis.

Recently, a novel family of proteins has been described in mammalians, characterized by a series of distinctive features: due to structural similarities with glutathione transferases 
(GST), they have been included in a new family, termed GST-omega (GSTO) (7). Despite belonging to the GSTs superfamily these proteins lack any appreciable glutathione transferase activity, but show a series of properties that indicate them as stress response proteins potentially involved in cancer progression: indeed, besides localizing into the cell nucleus after thermal damage in rodents $(8)$, they i) induce resistance against anticancer drugs and ionizing radiations (8); ii) reduce the oxidized form of vitamin $C$, dehydroascorbic acid, to the native form, ascorbic acid, thus involving them in the antioxidant defense mechanisms $(7,9-12)$, iv) catalyse thioltrasferase reaction $(7,11)$ and may reduce protein disulfides, thus contributing to the recovery from oxidative challenge to proteins (13). Polymorphisms of the two GSTO genes found in humans (GSTO1, GSTO2) have been associated with the risk of hepatocellular carcinoma, cholangiocarcinoma, breast cancer and ovarian cancer (14-16).

The present study was aimed to ascertain whether human GSTO1 retains the stress response properties found in rodents (8), and whether the activation of the human GSTO1 stress response, i.e., its nuclear translocation might serve as a progression marker of $\mathrm{BE}$ towards $\mathrm{NiN}$ and adenocarcinoma.

\section{Materials and methods}

Patients with diagnosis of Barrett's esophagus were identified from a database of Department of Medical and Surgical Gastroenterology spanning of a 2-year period (2004-2005).

This approach was necessary to compare two consecutive groups with intestinal metaplasia, one without non-invasive neoplasia $(\mathrm{NiN})$ (22 patients), and the other with different grade of NiN (22 patients). For the sake of comparison, two esophageal adenocarcinomas were also included in the study. Patients with previous history of Barret's disease were excluded from the study.

Patient evaluation and bioptic procedures. All patients were evaluated with a detailed history before undergoing upper endoscopy: global health status, the presence or absence of typical or atypical GERD symptoms (predominant symptom) and previous drug consumption were investigated.

All endoscopies were performed by the same operator using standard diagnostic endoscopes. BE was defined as a detectable upward displacement of the squamo-columnar junction at endoscopy, confirmed by intestinal metaplasia with goblet cells at histology. Systematic 4-quadrant biopsy specimens at 2-cm intervals along the entire length of the Barrett's segment starting from the gastroesophageal junction were obtained with standard-size forceps. Agreement on the presence and extension of BE mucosa and the degree of esophagitis was obtained in all cases. The esophagitis, if present, was classified into four grades (A through D) according to Los Angeles Classification (3).

All patients underwent gastric biopsies from antrum, greater curvature (two), and lesser curvature (two) for assessment of Helicobacter pylori (Hp) status; additional targeted biopsies were taken from erosions, nodules or ulcers. The esophageal biopsies specimen on Barrett's metaplasia were considered for this study.
Histology. All specimens were fixed in $10 \%$ formalin, embedded in paraffin, and cut into serial $4-\mu \mathrm{m}$-thick sections for hematoxylin-eosin, PAS-alcian blue (alcian blue at $\mathrm{pH} 2.5$, $1 \%$ periodic acid and Schiff's reagent), Giemsa stain, and for immunohistochemical evaluation.

Immunohistochemistry. Immunohistochemical studies were performed on sections placed on positively charged glass slides; after deparaffinization, the tissue sections were rehydrated, blocked for endogenous peroxidase activity with $3 \%$ hydrogen peroxidase in methanol $(10 \mathrm{~min})$, and washed under running water for $5 \mathrm{~min}$ and transferred to phosphatebuffered saline. The sections were then blocked with $2 \%$ horse serum for $15 \mathrm{~min}$. The slides were incubated for $30 \mathrm{~min}$ with a 1:1000 dilution of the primary rabbit anti-GSTO1 antibody. The sections were then incubated with the secondary antibody (Vectastain Elite ABC Kit, Vector Laboratories Inc., Burlington, CA) according to the manufacturer's instructions. The slides were washed with phosphate-buffered saline between incubations. The tissue sections were developed using aminoethyl carbazole as the chromogen and counterstained with hematoxylin. All immunohistochemical stains were evaluated for the presence, extent and location of GSTO expression.

Pathological evaluation of biopsies. All biopsies were evaluated by the same pathologist. Histological slices were re-evaluated in a blinded manner by the same pathologist to reduce the risk of intra-observer variability. According to Sampliner's criteria (4) BE was classified as the presence of specialized intestinal-type metaplasia with goblet cells. Noninvasive neoplasia $(\mathrm{NiN})$ was evaluated using the criteria of Rydell (17) and classified in three different groups: negative, indefinite and positive. Low-grade $\mathrm{NiN}$ and high-grade $\mathrm{NiN}$ represent two different subsets of the positive group: LG-NiN is defined by the presence of nuclear atypia involving the mucosal surface, nuclear stratification in the base of crypts, and preserved crypt architecture; HG-NiN is defined by the presence of marked nuclear atypia, distorted crypt architecture and nuclear stratification extending to the luminal surface.

Cell culture and heat shock experiments. HeLa cells were grown in D-MEM (Sigma) supplemented with 5\% heat inactivated serum, $1 \%$ L-Glutamine, in a humidified atmosphere of $5 \% \mathrm{CO}_{2}$, at $37^{\circ} \mathrm{C}$. For the heat shock experiments HeLa cells were incubated for 60,120 and $180 \mathrm{~min}$ at $43^{\circ} \mathrm{C}$ in a humidified atmosphere of $5 \% \mathrm{CO}_{2}$. HeLa cells at various time after heat shock treatment were washed in PBS, scraped and centrifuged at $1000 \mathrm{~g}$ for $2 \mathrm{~min}$. The cell pellet was washed with $500 \mu \mathrm{l}$ of buffer B (HEPES $10 \mathrm{mM} \mathrm{pH} \mathrm{7.9,} \mathrm{KCl}$ $10 \mathrm{mM}, \mathrm{MgCl}_{2} 2 \mathrm{mM}$, EDTA $\left.200 \mu \mathrm{M}\right)$, centrifuged at $1000 \mathrm{~g}$ 2 min and lysed in buffer B with triton X100 1\%, leupeptin $4 \mu \mathrm{g} / \mathrm{ml}$ and aprotinin $1 \mu \mathrm{g} / \mathrm{ml}$ on ice for $30 \mathrm{~min}$. The cell suspension was then centrifuged at $3000 \mathrm{~g}$ at $4^{\circ} \mathrm{C}$ for $5 \mathrm{~min}$, and the nuclear pellet was recovered and further washed with PBS. The nuclear fraction was stored at $-20^{\circ} \mathrm{C}$ unitl used.

SDS-PAGE electrophoresis and immunoblot. Sodium dodecylsulphate-polyacrylamide gel electrophoresis was 
performed essentially by the method of Laemmli (18), with 5\% acrylamide stacking gel and 15\% separating gel. Immunoblot was performed by transferring proteins to nitrocellulose sheets (Bio-Rad Laboratories) following the methods of Towbin et al (19). Immunodetection was performed employing anti-GSTO1 serum and was revealed by peroxidase-labeled anti-rabbit IgG (Sigma) and BM Chemiluminescence Blotting Substrate (POD) (Roche).

Immunofluorescence microscopy. HeLa cells were fixed in $4 \%$ paraformaldehyde for $20 \mathrm{~min}$ on ice; at the end of incubation paraformaldehyde was removed and the cells were washed in PBS. For the blocking step the cells were incubated in $8 \%$ milk with $1 \%$ Triton X-100 in PBS for 30 min at room temperature. Then the cells were incubated for $60 \mathrm{~min}$ at room temperature with anti-GSTO1 serum diluted 1:100 in $0.8 \%$ milk and $0.1 \%$ Triton $\mathrm{X}-100$. After rinsing twice in PBS, the cells were incubated for 30 min with the secondary anti-rabbit fluorescein labelled antibody (Sigma) diluted 1:100 in PBS. Images were captured using a Leica DM microscope (Leica, Germany).

Protein determination. Protein concentrations were determined using BCA method (Sigma) following the manufacturer's instruction.

Statistical analysis. Variables corresponding to the percentage of GSTO1-positive cells $(+,++$ or +++ , corresponding to $<25,<50$ or $>50 \%$ ), and to the prevalent subcellular localyzation of GSTO1 staining (nuclear, cytoplasmic or diffuse) in Barrett's epithelium were analyzed by the $\chi^{2}$ test (GraphPad Prizm Software).

\section{Results}

Subjects. Forty-six patients, 33 male and 12 female, with a mean age of $62.8( \pm 14)$ were included into the study. The predominant symptoms recorded before diagnostic endoscopy were: heartburn in $24 / 46(52.2 \%)$ patients, regurgitation in $11 / 46(23.9 \%)$, non-cardiac chest pain in $5 / 46(10.9 \%)$, epigastric pain in $3 / 46(6.5 \%)$, chronic cough in $2 / 46(4.3 \%)$ and dysphagia in $1 / 46(2.2 \%)$.

Endoscopic findings detected in the selected patients at the time of diagnosis were: absence of esophageal flogosis in $32 / 46(69.6 \%)$ patients, from A to D grade of esophageal flogosis in 12/46 (26.1\%) and AC in 2/46 (4.3\%).

Short-segment Barrett's esophagus $(\mathrm{SSBE})(<3 \mathrm{~cm})$ was detected in 28/44 (63.6\%) and long-segment Barrett's esophagus (LSBE) $(>3 \mathrm{~cm})$ in $16 / 44(36.4 \%)$. Among the selected patients with BE (44/46) NiN was absent in 22/44 (50\%), low-grade NiN (LG-NiN) was present in $7 / 44$ $(15.9 \%)$, high-grade NiN (HG-NiN) in 9/44 (20.5\%) and indefinite grade of $\mathrm{NiN}$ in $6 / 44(13.6 \%)$.

Infection with $H$. pylori was histologically detected in gastric biopsies in $8 / 45(17.8 \%)$ of selected patients. No previous treatment with proton pump inhibitors (PPI) or histamine $\mathrm{H} 2$ receptor antagonists had been performed within the previous year in all patients.

Immunohistochemistry of GSTO1. In the 46 biopsies of the lower esophagus studied, immunohistochemically detectable
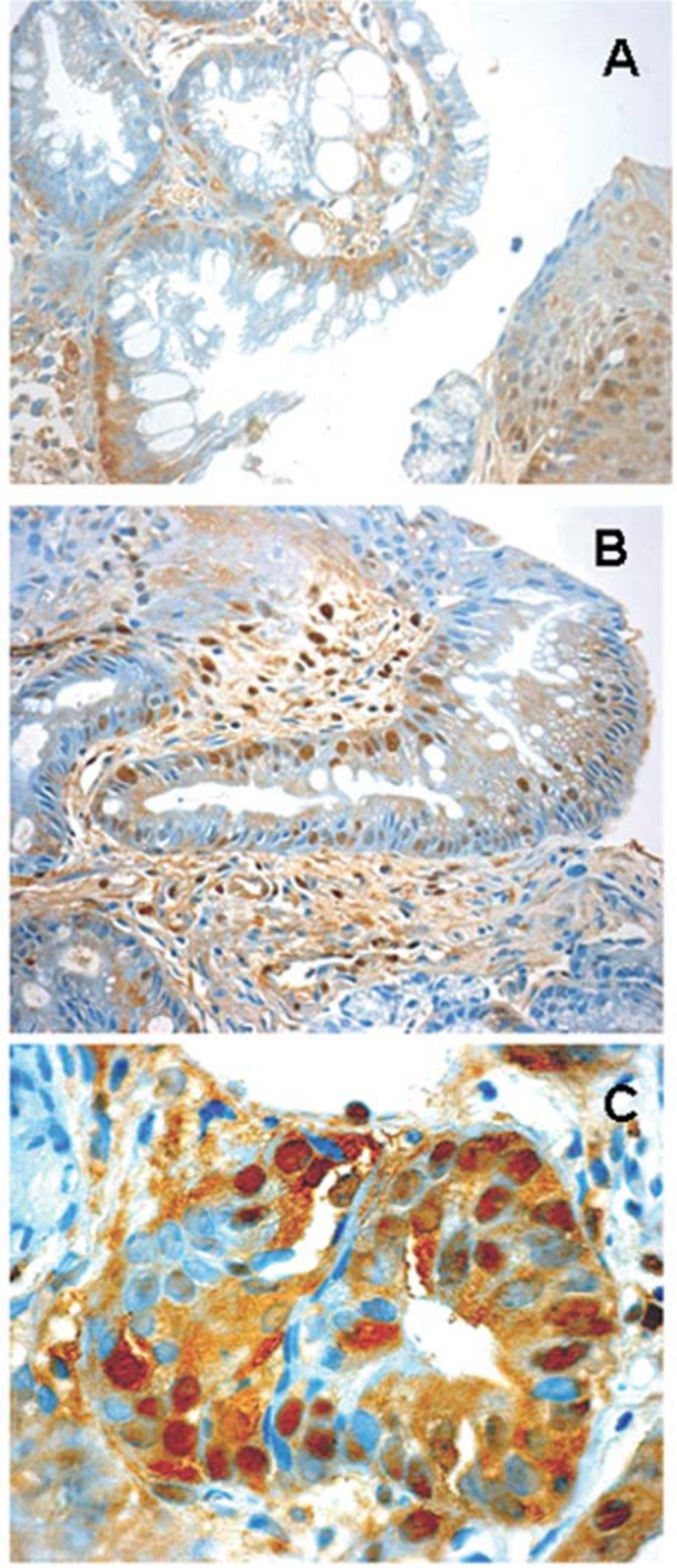

Figure 1. Immunolocalisation of GSTO1 in esophageal biopsies of subjects with Barrett's disease. Counterstaining: haematoxylin. (A) Barrett's esophagus without NiN. GSTO1 stainining involves the cytoplasm of the metaplastic cells and appears to be more intense in the basal portion of the cells, while most nuclei stain negative. Original magnification $x 400$. (B) Low-grade NiN, many dysplastic cells show a selective nuclear staining for GSTO1. Original magnification $\mathrm{x} 400$. (C) High-grade NiN, glandular acinus with intense and selective nuclear staining in most cells. Original magnification $\mathrm{x} 1000$.

GSTO1 was found to be localized in the surrounding esophageal epithelium, cardial and fundic mucosa, and diffuse low-grade staining was found in the mesenchyma between the glands (Fig. 1A and B). Particularly intense staining was found in endothelial cells. Among the 44 patients with BE, absence of $\mathrm{NiN}$ was found in 22, while 7 showed low-grade $\mathrm{NiN}$, and 9 high-grade NiN; 6 were indefinite for NiN. 

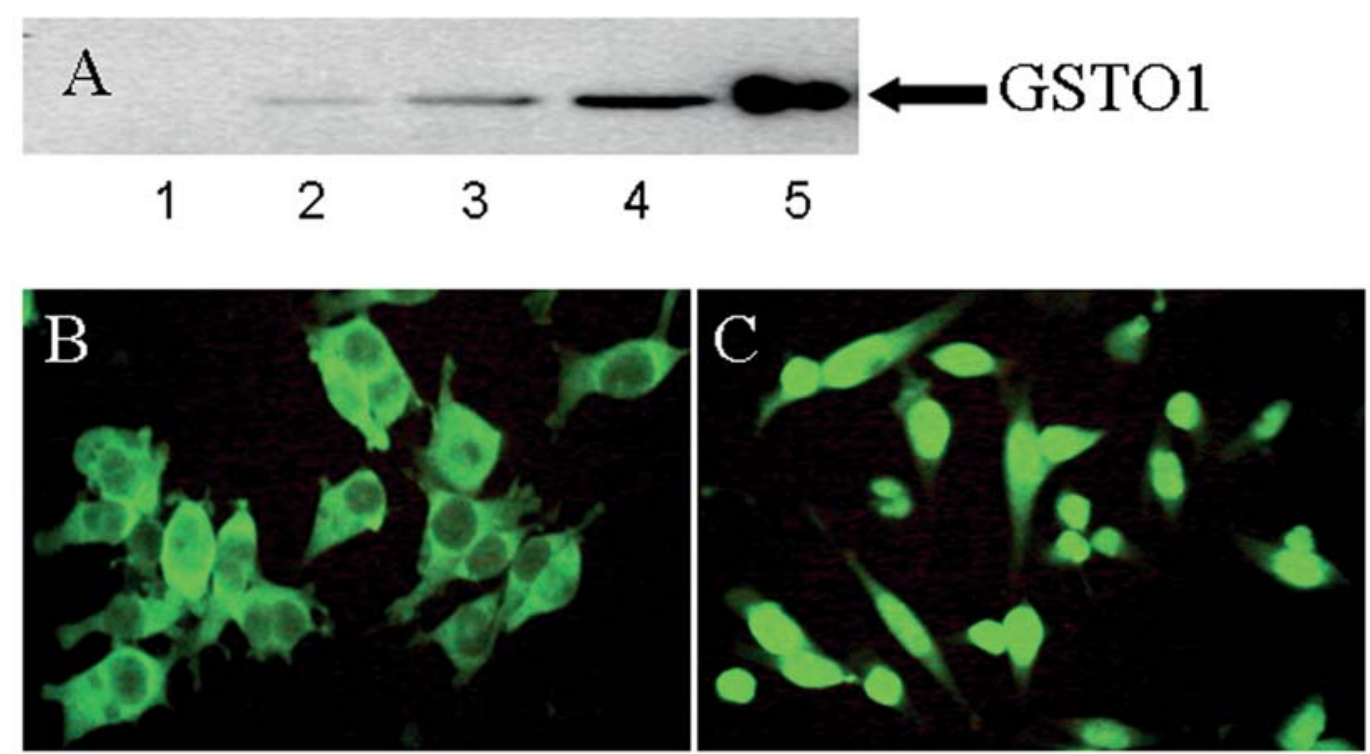

Figure 2. Effect of heat shock treatment at $43^{\circ} \mathrm{C}$ on subcellular localization of GSTO1 in HeLa cells. In (A) nuclear extracts of Hela cells at different time intervals after initiation of thermal shock lane 1, control culture; lane 2, $60 \mathrm{~min}$; lane 3, $120 \mathrm{~min}$; lane 4, $180 \mathrm{~min}$; Lane 5 human recombinant GSTO1. In (B) and (C), immunofluorescence of GSTO1 before and after 180 min of heath shock, respectively.

Among the 22 cases of Barrett's disease without NiN, $5 / 22$ cases had $<25 \%$ cells $(+)$ stained positive for GSTO, $9 / 22$ had $50 \%(++)$ and $8>50 \%$ positive cells $(+++)$. Among these cases without NiN, 4 showed prevalent nuclear localization, 13 cytoplasmic localization (Fig. 1A), and 5 diffuse cellular staining; no correlation was found between the proportion of cells stained and the localization of the staining. Out of the 22 cases with $\mathrm{NiN}$, all the 7 cases (4+ and 3++) with low-grade $\mathrm{NiN}$ and the 9 cases with high-grade $\mathrm{NiN}$ (5++ and 4+++) showed prevalent nuclear localization of GSTO1 immunostaining (Fig. 1B and C). The prevalence of nuclear localization of GSTO1 among the cases with $\mathrm{NiN}$ was significantly higher than among cases without $\mathrm{NiN}$ $(\mathrm{P}<0.0001)$. Out of the 6 cases not determined for $\mathrm{NiN}, 2$ showed nuclear, 2 cytoplasmic and 2 diffuse staining. Of the 2 cases of adenocarcinoma of the esophagus, one case $(+++)$ showed prevalent nuclear localization, and the other showed prevalent nuclear localization in the more differentiated areas $(+++)$, and diffuse staining in the less differentiated $(++)$.

Heat shock treatment. To investigate whether in human cells GSTO1 maintains the shock protein properties found in mice, HeLa cells, that were found to express GSTO1, were subjected to thermal stress at $43^{\circ} \mathrm{C}$ for different times. The nuclear translocation of immunoreactive protein to the nucleus started at the end of the first hour of incubation and reached the maximum level in $3 \mathrm{~h}$ (Fig. 2A). The nuclear translocation of GSTO1 was confirmed by immunofluorescence studies that showed the negative image of the nucleus in control cells (Fig. 2B), and the prevalent nuclear positivity after thermal shock (Fig. 2C).

\section{Discussion}

The glutathione transferases omega (GSTOs) are a new class of glutathione transferase that show a series of distinctive features which could make them relevant for cancer progression. In mice GSTO1 acts as a shock protein, translocating into the nucleus as a consequence of thermal stress, while in murine leukemia cells its overexpression was found to associate with increased resistance to ionizing radiations (8). Incresed expression of GSTO was found also in cisplatinresistant ovarian cancer SKOV3 cells (20) and polymorphisms of the two GSTO genes found in humans (GSTO1, GSTO2) have been associated with the risk of hepatocellular carcinoma, cholangiocarcinoma, breast cancer and ovarian cancer $(15,16)$.

In Barrett's esophagus the progression to invasive AC may develop through stages from metaplasia to increasing grade of NiN. Esophageal carcinogenesis is thus a multistep process of genetic instability due to the accumulation of the successive mutations in the columnar intestinal epithelial cells of BE, that lead to NiN and eventually to the cancer (5).

In the present work we found a selective nuclear localization of GSTO1 in all biopsies with NiN, while diffuse or selective cytoplasmatic staining was found only in most BE biopsies without signs NiN. Our main finding was thus that the selective nuclear translocation of GSTO1 is a marker of $\mathrm{NiN}$ in Barrett's esophagus, suggesting a possible use of GSTO1 immunostaining for improving the diagnosis of $\mathrm{NiN}$ in BE. In addition we found, for the first time, that human GSTO1 translocates into the nucleus as a consequence of heath shock in human cancer cells. Although the biological consequences of the nuclear translocation of GSTO1 in Barrett cells are unknown, this finding supports the hypothesis that the nuclear translocaton of GSTO1 is involved in the mechanisms of adaptation of dysplastic cells to the hostile environment of the lower esophagus, thus participating to the neoplastic progression towards esophageal cancer.

The involvement of GSTO1 translocation could extend beyond the simple adaptation to dysplastic environment, in fact among the several functions of GSTO1 ascertained until now, some could directly influence the mechanisms controlling cell proliferation and differentiation, in particular the 
thioltransferase activity of GSTO1 $(8,11)$ might be involved in the control of redox-regulated transcription factors or signalling elements, such as AP-1 or NF- $\mathrm{B}$, a function already demonstrated for other proteins such as thioredoxin $(21,22)$. In view of this, understanding the functional effects of the polymorphism found to be associated with increased susceptibility to cancer could help in understanding the precise role of GSTOs in cancer progression.

In conclusion, the nuclear translocation of GSTO1 in Barrett's NiN suggests a specific function of human GSTO1 in the progression of esophageal cancer. Although further studies are needed to establish the precise biological relevance of this phenomenon and the functions of the translocated protein, the subcellular localization of GSTO1 in bioptic specimens could provide, in addition to P53 and Ki67, a useful immunohistochemical diagnostic tool to increase specificity and sensitivity of the diagnosis of $\mathrm{NiN}$ in the Barrett's disease.

\section{Acknowledgements}

This study was supported by a 2007 PRIN grant of the Italian Ministry of University.

\section{References}

1. Winters C Jr, Spurling TJ, Chobanian SJ, et al: Barrett's esophagus. A prevalent, occult complication of gastroesophageal reflux disease. Gastroenterology 92: 118-124, 1987.

2. Csendes A, Smok G, Burdiles P, Korn O, Gradiz M, Rojas J and Recio M: Prevalence of intestinal metaplasia according to the length of the specialized columnar epithelium lining the distal esophagus in patients with gastroesophageal reflux. Dis Esophagus 16: 24-28, 2003.

3. Falk JW: Barrett's esophagus. Gastroenterology 122: 15691591, 2002.

4. Cameron AJ, Lomboy CT, Pera M and Carpenter HA: Adenocarcinoma of the esophagogastric junction and Barrett's esophagus. Gastroenterology 109: 1541-1546, 1995.

5. Pera M, Cameron AJ, Trastek VF, et al: Increasing incidence of adenocarcinoma of the esophagus and esophagogastric junction. Gastroenterology 104: 510-513, 1993.

6. Cameron AJ, Ott BJ and Payne WS: The incidence of adenocarcinoma in columnar-lined (Barrett's) esophagus. N Engl J Med 313: 857-859, 1985.

7. Board PG, Coggan M, Chelvanayagam G, Easteal S, Jermiin LS, Schlte GK, Danley DE, Hoth LR, Griffor MC, Kamath AV, Rosner MH, Chrunyk BA, Perreagaux DE, Gabel CA, Georghean FK and Pandit J: Identification, characterization, and crystal structures of the Omega Class Glutathione Trasferases. J Biol Chem 275: 24798-24806, 2000.
8. Kodym R, Calins P and Story M: The cloning and characterization of a new stress response protein a mammalian member of a family of teta class glutathone S-transferase-like protein. J Biol Chem 274: 5131-5137, 1999.

9. Maellaro E, Del Bello B, Sugherini L, Santucci A, Comporti M and Casini AF: Purification and caracterization of glutathionedependent dehydroascorbate reductase from rat liver. Biochem $\mathrm{J}$ 301: 471-476, 1994.

10. Xu DP, Washburn MP, Sun GP and Wells WW: Purification and caracterization of a glutathione-dependent dehydroascorbate reductase from human erithrocytes. Biochem Biophys Res Commun 221: 117-121, 1996.

11. Schmuck EM, Board PG, Whitbread AK, Tetlow N, Blackburn AC and Masoumi A: Characterization of the monomethylarsonate reductase and dehydroascorbate reductase activity of Omega class glutathione transferase variant. Pharmcogenet Genomics 15: 493-501, 2005.

12. Ishikawa KA, Casini AF and Nishikimi M: Molecular cloning and functional expression of rat liver glutathione-dependent dehydroascorbate reductase. J Biol Chem 273: 28708-28712, 1998.

13. Whitbread AK, Masoumi A, Tetlow N, Schmuck E, Coggan M and Board PG: Characterizatio of the omega class of glutathione transferases. Methods Enzymol 401: 78-102, 2005.

14. Whitbread AK, Tetlow N, Eyre HJ, Sutherland GR and Board PG: Characterizathion of human Omega class gluthathione transferase genes and associated polymorphisms. Pharmacogenetics 13: 131-144, 2003.

15. Marahatta SB, Punyarit P, Bhudisawasdi V, Paupairoj A, Wongkham S and Petmitr S: Polymorphism of glutathione Stransferase Omega gene and risck of cancer. Cancer Lett 236: 276-281, 2006.

16. Pangstaporn W, Rochanawutanon M, Wilailak S, Linasamita V, Weerakiat $\mathrm{S}$ and Petmitr S: Genetic alteration in chromosome 10q24.3 and glutathione S-transferase Omega 2 polymorphism in ovarian cancer. J Exp Clin Cancer Res 25: 107-114, 2006.

17. Spechler SJ, Robbins AH, Rubins HB, Vincent ME, Heeren T, Doos WG, Colton T and Schimmel EM: Adenocarcinoma and Barrett's esophagus. An overrated risk? Gastroenterology 87: 927933, 1984

18. Laemmli UK: Cleavage of structural proteins during the assembly of the head of bacteriophage T4. Nature 227: 680-685, 1970.

19. Towbin H, Stachelin P and Gordon J: Electrophoretic transfer of protein from polyacrylamide gels to nitrocellulose sheets: procedure and some applications. Proc Natl Acad Sci USA 76: 4350-4354, 1976.

20. Yan XD, Pan LY, Yuan Y, Lang JH and Mao N: Identification of platinum-resistance associated proteins through proteomic analysis of human ovarian cancer cells and their platinumresistant sublines. J Proteome Res 6: 772-780, 2007.

21. Weichsel A, Casdaska JR, Powis G and Montfort WR: Crystal structures of reduced, oxidized, and mutated human thiredoxin: evidence for a regulatory homodimer. Structures 4: 735-751, 1996.

22. Arner ES and Holmgren A: Physiological functions of thioredoxin and thioredoxin reductase. Eur J Biochem 267: 6102-6109, 2000 